\title{
RESPUESTA ECO-HIDROLÓGICA DE LOS SUELOS EN CAMPOS ABANDONADOS (SUR DE ESPAÑA)
}

\author{
J. D. RUIZ SINOGA* , J. F. MARTÍNEZ MURILLO \\ Departamento de Geografía, Universidad de Málaga. \\ Campus de Teatinos s/n. 29071. Málaga, España.
}

\begin{abstract}
RESUMEN. El proceso de abandono del campo en zonas marginales por la entrada en crisis de la actividad agrícola, y de la economía natural agraria, ha constituido un verdadero cambio de uso del suelo a gran escala. Los efectos del abandono del campo sobre la calidad de las tierras son muy diversos, dependiendo de las condiciones climáticas y del estado de los suelos, muy variable desde el punto de vista espacio-temporal. Así, suelos bajo condiciones climáticas favorables, con cubierta vegetal, pueden regenerarse con el tiempo por la acumulación de material orgánico e incremento de la actividad floral y de la fauna, mejorando su estructura, aumentando la infiltración del agua y, por tanto, decreciendo la erosión hídrica potencial.

El propósito de este trabajo fue caracterizar el patrón de vegetación de varios campos abandonados hace más de 50 años, localizados en las Cordilleras Béticas Internas sobre relieves de litologías metamórficas, así como la respuesta hidrológica de sus suelos mediante el seguimiento espacio-temporal de la humedad y la aplicación de lluvia simulada, haciendo especial énfasis en el efecto ocasionado por sus condiciones superficiales.

Los resultados han mostrado como las condiciones superficiales de sus suelos están indicando estadios diferentes en la evolución de éstos después del abandono de la actividad agrícola: los campos con más abundantes lluvias han alcanzado un equilibrio entre sus condiciones climáticas, topográficas y erosivas, mientras que los que están en condiciones más semiáridas aún se encuentran en una fase anterior donde la erosión hídrica puede presentar una elevada actividad durante los escasos eventos lluviosos.
\end{abstract}

\section{Eco-hydrological response of soils in abandoned fields}

ABSTRACT. The process of field abandonment in marginal areas by the entry of agricultural activity, and of the "agricultural natural economy" crisis, has been a real change of land use on a large scale. The effects of the abandonment of the field about the quality of the land vary widely, depending on weather conditions 
and the state of soils, highly variable from the spatio-temporal standpoint. Thus, soils under favourable climatic conditions, with vegetation cover, can regenerate over time by the accumulation of organic material and increase their biological activity and fauna, improving its structure, increasing infiltration of water and, therefore, decreasing the potential water erosion.

The purpose of this work was to characterize the vegetation pattern of several abandoned fields for more than 50 years, located in the internal Bethics mountain ranges on lithology of metamorphic reliefs, as well as the hydrological response from their soils by tracking spatio-temporal of the soil moisture and the application of simulated rain, with special emphasis on the effect caused by their surface conditions.

The results have shown as surface conditions from their soils are indicating different stages in the evolution of these after the abandonment of agricultural activity: the fields with more abundant rains have struck a balance between their conditions climatic, topographical and erosive, while those who are in most semi-arid conditions still are at an earlier stage where water erosion can present a high activity during rare rain events.

Palabras clave: abandono, resiliencia, degradación de suelos, hidrología superficial, erosión.

Key words: field abandonment, resilience, soil degradation, soil hydrology, water erosion.

Enviado el 29 de agosto de 2011 Aceptado el 28 de noviembre de 2011

* Correspondencia: Departamento de Geografía, Universidad de Málaga. Campus de Teatinos, s/n. 29071. Málaga, España. E-mail: sinoga@uma.es

\section{Introducción}

La región mediterránea es una de aquellas del planeta en las que los cambios climáticos y geomorfológicos, así como el impacto de la actividad humana han ocasionado una progresiva degradación medioambiental, finalizando en procesos de desertificación los cuales son especialmente graves en áreas de elevado estrés (regiones áridas y semiáridas) debido a su elevada vulnerabilidad (López Bermúdez, 1993).

Desde finales del siglo XIX, y más recientemente, a mediados del siglo XX, ha tenido lugar un proceso casi continuo de abandono del campo en zonas marginales por la entrada en crisis de la actividad agrícola, y de la economía natural agraria (Naredo, 1996), constituyendo un verdadero cambio de uso del suelo a gran escala. Los efectos del abandono del campo sobre la calidad de las tierras son muy diversos, dependiendo de las condiciones climáticas y del estado de los suelos, muy variable desde el punto de vista espacio-temporal. Los suelos bajo condiciones climáticas favorables, que sostienen la cubierta vegetal, pueden regenerarse con el tiempo por la acumulación de material orgánico e incremento de la actividad floral y de la fauna, mejorando su estructura, 
aumentando la infiltración del agua y, por tanto, decreciendo la erosión hídrica potencial. Estos rasgos pueden jugar un papel más o menos importante dependiendo de las características ecogeomorfológicas del lugar y se genera una gran variedad de situaciones, de ahí la dificultad de predecir la evolución futura de los campos abandonados (Kosmas et al., 2000).

Sí se ha demostrado que el abandono de los campos juega un papel importante en la restauración de la cubierta vegetal (Obando, 2002; Descroix y Gautier, 2002). Ruiz Flaño (1993) apunta que es la edad del abandono y el tipo de sucesión vegetal los factores más determinantes para explicar la dinámica de micro-ambientes en campos abandonados. Es obvio que un cambio de uso del suelo, de la magnitud con la que se ha producido en muchas áreas rurales mediterráneas, debe tener unos efectos transcendentales sobre la infiltración, la escorrentía y los aportes de sedimentos, resultando en cambios globales de la hidrología y de las tasas erosivas de los suelos (García Ruiz et al., 1995; Lasanta et al., 2000), con consecuencias impredecibles a escala de ladera y de cuenca (Lasanta et $a l ., 1995)$, por lo que es muy importante llevar a cabo políticas y técnicas para la ordenación de los recursos naturales y agronómicos a fin de evitar procesos degenerativos.

López Bermúdez et al. (1998) observaron que la recolonización de vegetación natural se producía adecuadamente en campos abandonados, tras el cambio de uso del suelo, al mismo tiempo, que determinaron diferencias estructurales y funcionales entre la vegetación natural del campo abandonado y el matorral. La dinámica de ambas es igualmente diferente, con la evolución estacional de la producción de materia orgánica y la cubierta vegetal, mostrando una gran dependencia de la humedad del suelo para la vegetación del campo abandonado. Kosmas et al. (2002) apuntaron que la evolución del tipo de vegetación en campos abandonados del medio mediterráneo, mostraba una clara tendencia: predominio de plantas anuales durante los primeros cinco años y, tras este período, un progresivo decrecimiento de estas, al mismo tiempo que los matorrales aumentaban su presencia de forma progresiva. Algo similar encuentran Ruiz Sinoga y Martinez Murillo (2009), al analizar los componentes superficiales del suelo de un campo abandonado y su comportamiento hidrológico.

Tras el uso humano del suelo, el sistema natural puede recuperar las condiciones previas a la actividad, iniciándose procesos de recuperación de la cubierta vegetal como acabamos de apuntar; esta capacidad de recuperación ha sido denominada como resilencia. Dentro del paisaje mediterráneo, el suelo y las condiciones hidrológicas, sobre todo la disponibilidad de agua, importantes para la recuperación de la vegetación, varían espacialmente. A causa de esta falta de uniformidad del paisaje, es poco común que la resilencia del geo-ecosistema sea continua, por lo que es más que posible que coexistan unidades fisiográficas en proceso de degradación y otras en proceso de recuperación. El geo-sistema parece tener una capacidad de resilencia mayor si la exposición es a umbría que a solana, a escala de ladera. En las zonas más degradadas, la resilencia es mayor en la sección baja de la ladera porque la distancia a los recursos hídricos así como la incidencia de la radiación solar, que controla la evapotranspiración, es menor (Bergkamp, 1998).

A una escala espacial amplia, la resilencia está muy influenciada por la contribución de pequeñas áreas sensibles desde un punto de vista ecológico. Desde el punto de vista de 
la degradación de tierras, la resilencia de un lugar es importante en relación al tiempo necesitado (después de una perturbación) para que el suelo y la vegetación recuperen su papel protector contra las fuerzas que causan la degradación y la erosión. Se dice entonces que un área es sensible cuando su resilencia es baja y su degradación puede llegar a afectar al funcionamiento normal de áreas adyacentes (Imeson y Cammeraat, 2002).

Muchas de estas áreas se encuentran actualmente ocupadas por vegetación natural dispuesta espacialmente a modo de patrón discontinuo o en dos fases, de zonas de vegetación (matorral, principalmente) y suelo desnudo. Han sido numerosos los estudios acerca de la respuesta hidrológica de sus suelos que se han llevado a cabo, estableciéndose en general un modelo de respuesta hidrológica basado en zonas contribuyentes de escorrentía y sedimentos (suelo desnudo) y zonas sumidero (matorral), generalmente realizados en áreas semiáridas de media montaña mediterránea con sustrato geológico calcáreo (Yair y Klein, 1973; Yair y Lavee, 1985; Yair y Enzel, 1987; Lavee et al., 1991; Boix et al., 1995; Cerdà et al., 1996; Nicolau et al., 1996; Imeson et al., 1998; Lavee et al., 1998; Bergkamp et al., 1999; Calvo et al., 2003; Arnau Rosalen et al., 2008). Otros autores observaron similares respuestas en suelos de laderas de relieves con sustratos geológicos metamórficos (Puigdefábregas et al., 1999; Martínez Murillo y Ruiz Sinoga, 2007).

Esta respuesta hidrológica descrita se encuentra controlada por una serie de factores, descritos ampliamente en la literatura científica: contenido de gravas, textura y estabilidad de agregados de los suelos (Boix Fayos, 1999; van Wesemael et al., 1996, Boix et al., 1998), cambios espaciales y temporales de la cubierta vegetal (Cerdà, 1998; Lavee et al., 1998; Calvo et al., 2003; Ruiz Sinoga et al., 2010), uso previo y manejo del suelo (Ruiz Flaño, 1993; Lasanta et al., 2000), humedad del suelo previa a la lluvia (Castillo et $a l ., 2003)$. A estos factores, debe añadirse la presencia de un elevado porcentaje de fragmentos rocosos en la superficie del suelo, resultantes de la degradación del suelo por el laboreo agrícola, el pastoreo y la erosión hídrica (Poesen y Lavee, 1994; Valentín, 1994; Poesen et al., 1999; Ruiz Sinoga y Martínez Murillo, 2009). En este sentido, es clave el tamaño y la disposición de los fragmentos rocosos respecto a la superficie del suelo (sobre la superficie o embebidos en ella formando una costra continua) lo cual puede controlar su respuesta hidrológica (Lavee y Poesen, 1991; Poesen y Ingelmo-Sánchez, 1992).

El propósito de este trabajo fue caracterizar el patrón de vegetación de varios campos abandonados hace más de 50 años, localizados en las Cordilleras Béticas Internas sobre relieves de litologías metamórficas, así como la respuesta hidrológica de sus suelos mediante el seguimiento espacio-temporal de la humedad y la aplicación de lluvia simulada, haciendo especial énfasis en el efecto ocasionado por sus condiciones superficiales.

\section{Materiales y métodos}

\section{1. Áreas experimentales}

Los campos abandonados que constituyen las áreas experimentales de este trabajo se encuentran localizados a lo largo de las Cordilleras Béticas Internas (fig. 1), donde puede observarse un fuerte gradiente climático desde el Estrecho de Gibraltar 


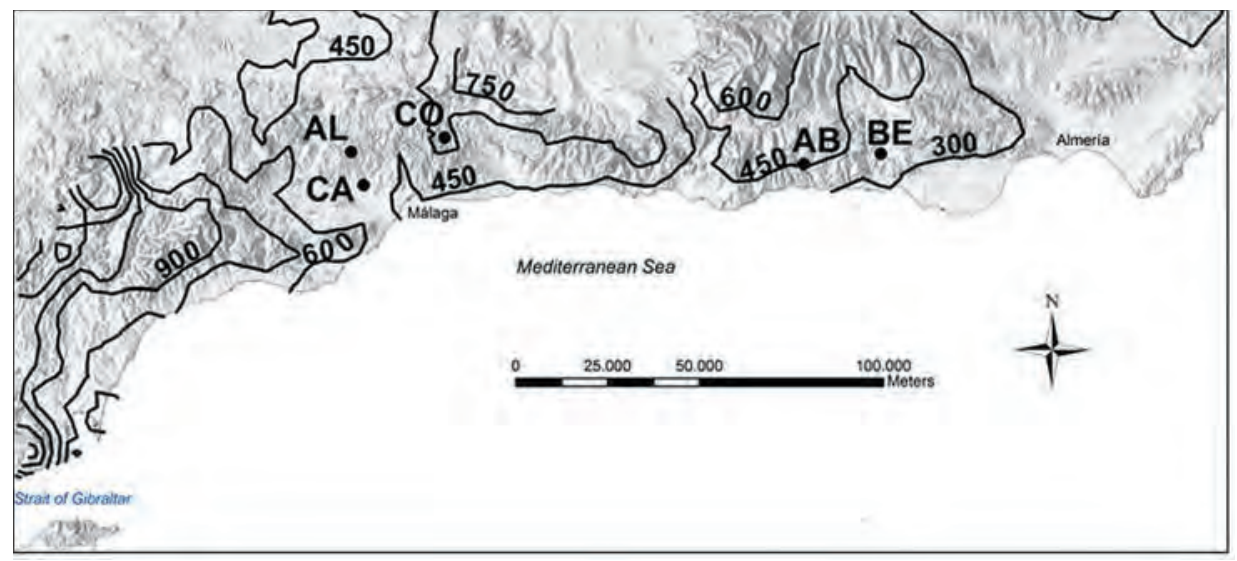

Figura 1. Localización de las áreas experimentales de campos abandonados. Abreviaturas: AL, Almogía; AB, Albuñol; BE, Berja; CA, Campanillas; CO, Colmenar.

(precipitación media anual $>1000 \mathrm{~mm} / \mathrm{año}$ ) hasta el Cabo de Gata (precipitación media anual $<250 \mathrm{~mm} / \mathrm{año}$ ). Estas áreas experimentales se seleccionaron siguiendo el método del gradiente climático propuesto por Imeson y Lavee (1998), de modo que los rasgos topográficos (pendiente, orientación y morfología), material parental (filitas y esquistos) y uso del suelo (campo abandonado) fueron similares para poder efectuar comparaciones de datos entre ellas. Las cinco áreas experimentales se encuentran en las provincias de Málaga (Montes de Málaga: Campanillas -CA-, Almogía-ALy Colmenar -CO-), Granada (Sierra de Contraviesa: Albuñol -AB-) y Almería (Sierra de Contraviesa: Berja -BE-).

En la tabla 1, se muestran las principales características ecogeomorfológicas de las laderas donde se sitúan los campos abandonados seleccionados. Los rasgos topográficos de las laderas de sustrato geológico metamórfico eran muy similares, con perfiles convexos. Todas las áreas experimentales estaban ya abandonadas en la década de los cincuenta según se observa en la fotografía aérea de 1956. Según fuentes de información local, el tipo de cultivo existente en estos campos era principalmente almendro o almendro+cereal. Este antiguo uso junto con las características topográficas, más las variables condiciones climáticas, ha favorecido la erosión de los suelos por parte del agua, los cuales han quedado bastante disminuidos a profundidades no superiores a $50 \mathrm{~cm}$ y con un elevado contenido de fragmentos rocosos en superficie (cubierta de 25 a $70 \%$; máxima en CA: $75 \%$ ) y en perfil (contenido en gravas $>55 \%$; máximo en CA: 72\%). De este modo, los suelos son eminentemente de tipo Leptosol y Regosol en las zonas bajas de las laderas. El contenido de arcillas del suelo fue siempre superior al 20\% en las áreas experimentales más lluviosas definiéndose una textura de tipo franco-arcillosa, mientras que dicho contenido se reducía en las áreas más secas, con texturas franco-arenosas en AB y BE. En cuanto al contenido de materia orgánica y la estabilidad de los agregados, se observó una reducción en ambos conforme la aridez se incrementaba. 
Tabla 1. Características eco-geomorfológicas de las áreas experimentales de campos abandonados. Abreviaturas: CO, Colmenar; AL, Almogía; CA, Campanillas; AB, Albuñol;

$B E$, Berja; $P$, precipitación media anual; M., mediterráneo; Pte., pendiente media;

E, exposición; L, longitud; L, litología; $G$, contenido de gravas; $A$, contenido de arcillas; $M O$, materia orgánica; Po, porosidad; EA, estabilidad de agregados; $C C$, contenido de agua en capacidad de campo; PMP, punto de marchitez permanente; $C V$, cubierta vegetal.

\begin{tabular}{|c|c|c|c|c|c|}
\hline $\begin{array}{l}\text { Área } \\
\text { experimental }\end{array}$ & $\mathrm{CO}$ & $\mathbf{A L}$ & $\mathrm{CA}$ & $\mathbf{A B}$ & BE \\
\hline P (mm/año) & 650 & 550 & 490 & 450 & 335 \\
\hline Clima & M. sub-húmedo & M. seco & M. semiárido & M. semiárido & M. semiárido \\
\hline Pte. $\left({ }^{\circ}\right)$ & 23.1 & 16.1 & 19 & 17.5 & 20 \\
\hline $\mathrm{E}\left({ }^{\circ}\right)$ & 178 & 175 & 170 & 180 & 180 \\
\hline $\mathrm{L}(\mathrm{m})$ & 130 & 90 & 125 & 83 & 65 \\
\hline $\mathrm{L}$ & Phyllites & Phyllites & Phyllites & Mica-schists & Mica-schists \\
\hline \multicolumn{6}{|c|}{ Propiedades edáficas } \\
\hline $\mathrm{G}(\%)$ & 59 & 60 & 70 & 48.1 & 51 \\
\hline $\mathrm{A}(\%)$ & 24 & 28 & 25 & 13.5 & 16.5 \\
\hline MO (\%) & 3.5 & 3.3 & 2 & 1.3 & 1.6 \\
\hline Po $(\%)$ & 41.9 & 43.1 & 42.3 & 47.2 & 42.7 \\
\hline EA $(\%)$ & 80 & 73 & 35 & 45 & 49 \\
\hline $\mathrm{CC}\left(\mathrm{cm}^{3} / \mathrm{cm}^{3}\right)$ & 0.23 & 0.22 & 0.14 & 0.20 & 0.23 \\
\hline $\operatorname{PMP}\left(\mathrm{cm}^{3} / \mathrm{cm}^{3}\right)$ & 0.08 & 0.07 & 0.05 & 0.02 & 0.03 \\
\hline $\begin{array}{l}\text { Principales } \\
\text { especies } \\
\text { vegetales }\end{array}$ & $\begin{array}{l}\text { Ulex baeticus, } \\
\text { Genista } \\
\text { umbellata, } \\
\text { Cistus albidus }\end{array}$ & $\begin{array}{c}\text { Cistus } \\
\text { monspelliensis, } \\
\text { Genista umbellata, } \\
\text { Helychrisum } \\
\text { stoechas }\end{array}$ & $\begin{array}{c}\text { Genista umbellata, } \\
\text { Retama } \\
\text { sphaerocarpa }\end{array}$ & $\begin{array}{l}\text { Genista umbellata, } \\
\text { Thymus vulgaris, } \\
\text { Thymalea } \\
\text { hirsuta }\end{array}$ & $\begin{array}{c}\text { Genista umbellata, } \\
\text { Thymalea } \\
\text { hirsuta, } \\
\text { Lavandaula } \\
\text { multifida }\end{array}$ \\
\hline CV $(\%)$ & 53.0 & 55.0 & 33.0 & 30.0 & 45.0 \\
\hline
\end{tabular}

Los cinco campos abandonados se encuentran recolonizados por vegetación natural en diferente grado, variando la cubierta vegetal entre 40 y $75 \%$ dependiendo de la estación del año. Esta vegetación está constituida por especies de matorral mediterráneo, siendo la más frecuente Genista umbellata dado que se observa su presencia en todas las laderas muestreadas. Conforme el clima se hace más húmedo, se observan nuevas especies vegetales con mayores necesidades hídricas: Cistus monspeliensis y Cistus albidus. Y lo contrario ocurre cuando las precipitaciones se hacen más escasas en AB y BE: Thymelaea hirsuta y Cistus clusii. Todos los campos abandonados seleccionados están afectados por pastoreo caprino, aunque no resulta una actividad intensiva dado que su paso por las laderas es bastante esporádico. 


\subsection{Caracterización del patrón de vegetación y las condiciones superficiales de los suelos}

La caracterización del patrón de vegetación y de las condiciones superficiales de los suelos en los campos abandonados se realizó mediante el método empleado ya por varios autores (Calvo et al., 2005; Arnau-Rosalén et al., 2008; Ruiz-Sinoga et al., 2010). Consiste en la delimitación de una parcela abierta con las siguientes dimensiones: 5 metros de ancho por toda la longitud de la ladera (desde la divisoria hasta el talweg). A su vez, esta parcela se subdivide en una retícula constituida por rectángulos de $2.5 \mathrm{~m} \mathrm{x}$ 3 , marcados con clavos en sus esquinas, georreferenciados mediante un GPS Leica 1200. Esta retícula se fotografía de modo aéreo usando una cámara digital, instalada en una estructura metálica de $4.75 \mathrm{~m}$ de altura, de modo perpendicular a la superficie del terreno. Las imágenes resultantes son georeferenciadas y rectificadas usando un sistema de información geográfica (ArcGIS 9.3).

La foto-interpretación de estas imágenes tiene en cuenta dos tipos de variables: abióticas (afloramientos rocosos, suelo desnudo, cubierta y disposición de fragmentos de rocas sobre y embebidos en la superficie del suelo y encostramientos) y bióticas (individuos de matorral, plantas muertas, vegetación anual, musgo y hojarasca). El resultado es un mapa del patrón espacial de vegetación y condiciones superficiales del suelo en las parcelas abiertas de los campos abandonados seleccionados; el procedimiento se repitió cada seis meses para evaluar los cambios estacionales en la cubierta vegetal en los campos abandonados de Colmenar, Almogía y Berja. La información obtenida durante este proceso permitió calcular la ratio \% cubierta vegetal $/ \%$ suelo desnudo.

\subsection{Caracterización hidrológica de los suelos}

La caracterización hidrológica de los suelos seleccionados se efectuó mediante dos métodos: uno, el seguimiento espacio-temporal de la humedad del suelo; y otro, la aplicación de lluvia simulada sobre individuos de matorral y las principales condiciones superficiales de los suelos.

El contenido de humedad del suelo se midió durante un periodo de dos años $(01 / 11 / 2002$ a 31/10/2004) en una serie de puntos fijos de muestro localizados a lo largo de la parcela abierta definida en las áreas experimentales de $\mathrm{CO}, \mathrm{AL}$ y $\mathrm{BE}$, dado que eran aquéllas entre las que había mayores diferencias climáticas. En cada punto de muestreo, se instaló una sonda en los primeros $15 \mathrm{~cm}$ de profundidad y cada semana se procedía a realizar una medición con un Tektronix $1502 \mathrm{C}$. El valor obtenido en la medición era transformado posteriormente a humedad volumétrica mediante la ecuación definida por Topp et al. (1980).

Las lluvias simuladas se llevaron a cabo sobre matorral y suelo desnudo, con diferentes condiciones superficiales. En total, se efectuaron 25 simulaciones lluvia (5 en cada área experimental) sobre un suelo en capacidad de campo. El simulador de lluvia empleado fue el diseñado por Calvo et al. (1988), posteriormente modificado por Cerdà et al. (1997), y los experimentos se realizaron sobre una parcela cerrada de $0.24 \mathrm{~m}^{2}$ ins- 
talada previamente en campo, con una salida orientada de modo paralelo a la máxima pendiente para la recolección de escorrentía y sedimentos. La lluvia simulada tuvo una duración equivalente al tiempo necesario hasta conseguir una tasa de escorrentía estable, el cual nunca excedió de 60 minutos. La intensidad de lluvia simulada fue aproximada de $40 \mathrm{~mm} \mathrm{~h}^{-1}$. La escorrentía se midió a intervalos de 1 minuto y cada cinco se tomó una muestra para la medición de los sedimentos en suspensión en laboratorio, mediante evaporación y secado en estufa a $105^{\circ} \mathrm{C}$. Los datos obtenidos en estos experimentos permitieron calcular las siguientes variables: tasa de infiltración, escorrentía total, coeficiente de escorrentía, concentración de sedimentos y tasa de erosión. También, el contenido de humedad del suelo en los primeros $5 \mathrm{~cm}$ fue medido antes y después de las simulaciones de lluvia. Por último, a los datos hidrológicos de las simulaciones de lluvia se le ajustó el modelo de Horton aplicando la ecuación (1):

$$
R=(I-F c)^{*}\left[1-\exp \cdot\left(-\alpha^{*}\left(T-60^{*} P o / I\right)\right)\right]
$$

Donde: $R\left(\mathrm{~mm} \mathrm{~h}^{-1}\right)$, escorrentía; $I\left(\mathrm{~mm} \mathrm{~h}^{-1}\right)$, intensidad de lluvia; $F c\left(\mathrm{~mm} \mathrm{~h}^{-1}\right)$, tasa de infiltración estable; $\alpha$, tasa de decaimiento de la tasa de infiltración o velocidad a la que Fc es alcanzada; $T$ ( $\mathrm{min}$ ), tiempo desde el comienzo de la lluvia; Po (mm), lluvia necesaria para el inicio de la escorrentía.

\section{Resultados y discusión}

\subsection{Patrón de vegetación y condiciones superficiales del suelo}

Los mapas resultantes de la fotointerpretación se muestran en la fig. 2, así como la vegetación y condiciones superficiales del suelo, en la tabla 2. Una de las principales diferencias post-abandono es la presencia o no de fragmentos rocosos embebidos en la superficie del suelo, formando una costra continua, y de vegetación terófita o herbáceas anuales. La cubierta vegetal no siguió una tendencia de decaimiento conforme se reducía la precipitación media anual. La vegetación está integrada por matorral de alta y baja densidad, es decir, individuos que forman una cobertura continua o discontinua sobre el suelo; también, fue posible cartografiar numerosos individuos de plantas muertas dado que la época del año era final de verano, momento en el que la cubierta vegetal está más reducida por la falta de agua en el suelo. En cuanto a la presencia de suelo desnudo, esto era común en todas las parcelas, aunque se observaron diferencias en cuanto a la cantidad de fragmentos rocosos sobre el suelo: mayor en AB y BE.

$\mathrm{Al}$ igual que la cubierta vegetal, la ratio vegetación/suelo desnudo no se redujo al disminuir la precipitación media anual. Ello parece indicar la presencia de factores limitantes de la recuperación de la cubierta vegetal tras el abandono de la actividad agrícola, especialmente, en las áreas experimentales más lluviosas. No obstante, las condiciones superficiales del suelo en los campos abandonados más secos parecen indicar unas peores condiciones ecogeomorfológicas y más propensas a favorecer procesos de erosión hídrica, dada la mayor presencia de zonas de suelo desnudo con pedregosidad superficial embebida. 


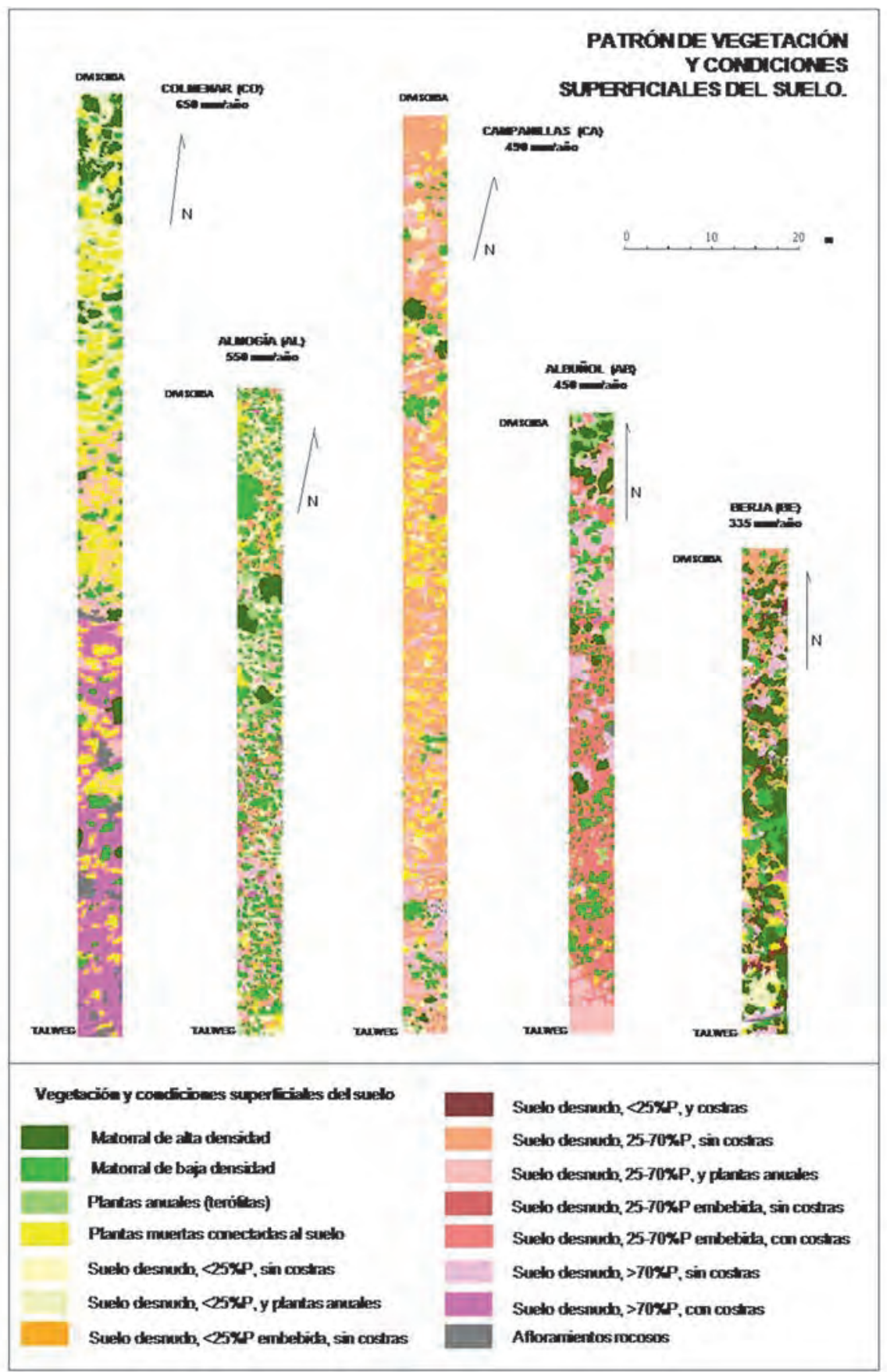

Figura 2. Mapas del patrón de vegetación y de las condiciones superficiales del suelo en las parcelas abiertas localizadas en los campos abandonados seleccionados. Abreviaturas: $P$, pedregosidad superficial. 
Tabla 2. Inventario y distribución porcentual de los elementos integrantes del patrón de vegetación y de las condiciones superficiales del suelo en las parcelas abiertas de los campos abandonados seleccionados. Abreviaturas: CO, Colmenar; AL, Almogía; CA, Campanillas; $A B$, Albuñol; BE, Berja; SD, suelo desnudo; $P$, pedregosidad superficial; $C V$, cubierta vegetal.

\begin{tabular}{|l|c|c|c|c|c|}
\hline Tipo de vegetación y condición superficial del suelo & CO & AL & CA & AB & BE \\
\hline Matorral de alta densidad (\%) & 7.7 & 12.4 & 1.8 & 9.3 & 15.6 \\
\hline Matorral de baja densidad (\%) & 19.3 & 33.4 & 7.1 & 18.4 & 25 \\
\hline Plantas anuales o terófitas (\%) & - & 4.2 & 11.3 & 0.9 & 0.8 \\
\hline Plantas muertas conectadas al suelo (\%) & 26.0 & 5 & 14.6 & 1.4 & 3.6 \\
\hline SD, <25\% P, sin costras (\%) & 3.1 & 13 & 5.7 & - & 0.9 \\
\hline SD, <25\% P, y plantas anuales (\%) & 12.7 & 8.2 & 0.4 & 3.2 & 3 \\
\hline SD, <25\% P, embebida, sin costras (\%) & - & 0.1 & - & - & - \\
\hline SD, 25-70\% P, sin costras (\%) & 1.4 & 15.5 & 48 & - & 17.5 \\
\hline SD, 25-70\% P, y plantas anuales (\%) & 10.4 & 10.3 & 15.1 & 16.8 & 4 \\
\hline SD, 25-70\% P, y costras (\%) & - & - & - & 39.7 & - \\
\hline SD, 25-70\% P, embebida y costras (\%) & 24.5 & 0.6 & - & - & - \\
\hline SD, >70\% P (\%) & - & 3 & 7.2 & 10.7 & 7.9 \\
\hline SD, >70\% P, embebida (\%) & - & 2.1 & - & - & 0.8 \\
\hline Afloramientos rocosos (\%) & 5.2 & 0.3 & - & 0.4 & 2.6 \\
\hline Cubierta vegetal (\%) & 53 & 55 & 33 & 30 & 45 \\
\hline SD total & 47 & 45 & 67 & 70 & 55 \\
\hline Ratio CV/SD & 1.13 & 1.22 & 0.49 & 0.43 & 0.82 \\
\hline
\end{tabular}

El patrón de vegetación y las condiciones superficiales de los suelos indican una cierta semejanza en los elementos que integran el sistema ecogeomorfológico de los campos abandonados seleccionados, independientemente de las condiciones climáticas puesto que se ha observado una similar variabilidad espacial en ellos. Así, todos los campos abandonados se encuentran caracterizados por un patrón de vegetación en bandas o de dos fases (matorral y suelo desnudo) como ha sido también observado por otros autores en condiciones mediterráneas (Puigdefábregas et al., 1998; Calvo Cases et al., 2005; Arnau Rosalén et al., 2008; Ruiz Sinoga et al., 2010). Estos mismos autores señalaban también las repercusiones hidrológicas y erosivas de este tipo de patrón al definir áreas fuente y sumidero de escorrentía y sedimentos. Sólo diferencias en las condiciones superficiales del suelo en cuanto a cantidad y disposición de la pedregosidad superficial del suelo hacen diferir unos campos de otros. 


\subsection{Respuesta hidrológica de los suelos}

Seguimiento de la humedad del suelo. Entre otros factores, el patrón de vegetación de una ladera incide en la variabilidad espacial y temporal de la humedad del suelo puesto que el crecimiento de las plantas depende de ello. En CO, AL y BE, durante un periodo de dos años se procedió a medir periódicamente la humedad del suelo de los primeros $15 \mathrm{~cm}$. La tabla 3 recoge información sobre este procedimiento experimental. Como es habitual en condiciones mediterráneas, la humedad del suelo siguió un patrón temporal semejante a la estacionalidad de las precipitaciones como puede observarse en la fig. 3. En CO, a pesar de ser el lugar más lluvioso, no registramos los valores más elevados, si bien en términos generales, en éste la humedad se mantuvo en niveles más altos durante las estaciones húmedas. Aunque durante el periodo de muestreo se registraron eventos de abundante precipitación, las medidas registradas no alcanzaron valores muy altos y los valores medios no superan los $0.10 \mathrm{~cm}^{3} \mathrm{~cm}^{-3}$, en lo que ha de tener gran influencia los valores tan bajos registrados durante el verano. Es destacable el fuerte descenso de la humedad del suelo cuando cesan las precipitaciones, traduciéndose en una severa sequía edáfica (contenido de humedad inferior al punto de marchitez permanente) de cuatro meses de duración en $\mathrm{CO}$ y AL, y de cinco a seis meses en BE.

Tabla 3. Datos relativos a la humedad del suelo $\left(\mathrm{cm}^{3} / \mathrm{cm}^{3}\right)$ medida en las parcelas de los campos abandonados de Colmenar (CO), Almogía (AL) y Berj a (BE). Abreviaturas: M., media;

Ds, desviación estándar; Máx, valor máximo registrado; Mín, valor mínimo registrado.

\begin{tabular}{|l|c|c|c|c|}
\hline \multicolumn{1}{|c|}{ Total } & M. & 0.07 & AL & BE \\
\cline { 2 - 5 } & Ds & 0.05 & 0.07 & 0.04 \\
\cline { 2 - 5 } & Máx & 0.27 & 0.04 & 0.03 \\
\cline { 2 - 5 } & Mín & 0.005 & 0.001 & 0.22 \\
\hline Sección superior & M. & 0.07 & 0.06 & 0.001 \\
\cline { 2 - 5 } & Ds & 0.04 & 0.04 & 0.04 \\
\cline { 2 - 5 } & Máx & 0.24 & 0.24 & 0.03 \\
\cline { 2 - 5 } & Mín & 0.005 & 0.001 & 0.001 \\
\hline \multirow{4}{*}{ Sección media } & M. & 0.07 & 0.08 & 0.05 \\
\cline { 2 - 5 } & Ds & 0.06 & 0.05 & 0.03 \\
\cline { 2 - 5 } & Máx & 0.27 & 0.26 & 0.20 \\
\cline { 2 - 5 } & Mín & 0.002 & 0.001 & 0.001 \\
\hline Sección baja & M. & 0.05 & 0.06 & 0.04 \\
\cline { 2 - 5 } & Ds & 0.05 & 0.04 & 0.04 \\
\cline { 2 - 5 } & Máx & 0.20 & 0.20 & 0.22 \\
\cline { 2 - 5 } & Mín & 0.001 & 0.001 & 0.001 \\
\hline
\end{tabular}



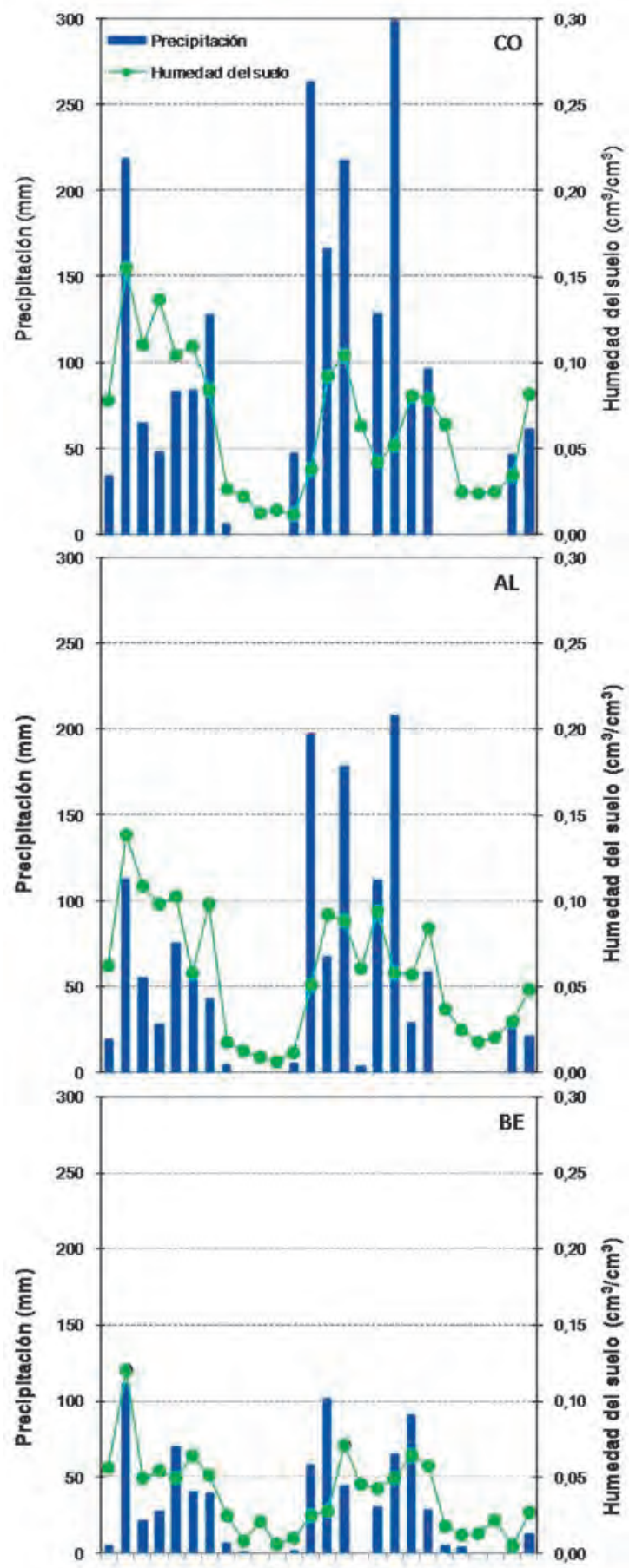

Figura 3. Variabilidad temporal de las precipitaciones y la humedad del suelo en los primeros $15 \mathrm{~cm}$ durante el periodo de muestreo en los campos abandonados de Colmenar (CO), Almogía (AL)

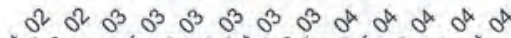

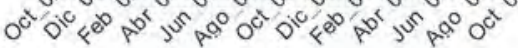
y $\operatorname{Berja}(B E)$. 
La distribución espacial de la humedad del suelo a lo largo de las parcelas experimentales se caracterizó por una gran heterogeneidad. En CO y AL, se observó una gran disparidad entre las diferentes secciones de las parcelas, mientras que en BE la uniformidad fue más evidente; la escasez de precipitaciones produce una homogeneización en la humedad superficial del suelo, sin que puedan entrar en juego otros factores bióticos y abióticos como en $\mathrm{CO}$ o AL. Tanto en $\mathrm{CO}$ como en AL, la mayor pendiente del terreno $(<25 \%)$ en la zona baja de la parcela experimental se tradujo en un decrecimiento en la humedad del suelo, al verse limitada la infiltración de agua. La misma relación con la topografía pero de modo inverso se observa en BE hacia la zona más alta de la ladera (pendiente $=28 \%$ ). De hecho, el coeficiente de correlación de Pearson corrobora de modo significativo esta relación entre pendiente y contenido superficial de humedad: $\mathrm{CO}, r=-0.68$; AL, $r=-0.56$; BE, $r=-0.53 ; p<0.05$.

Kosmas et al. (2000) indicaron que el clima y la degradación del suelo son factores claves en la recuperación de los campos abandonados, de modo que cubierta vegetal, capacidad de retención hídrica y humedad edáfica tienen una relación directa (Lavee et al., 1998). Dependiendo del grado de degradación del suelo, el sistema ecogeomorfológico se caracterizará por un nivel de resilencia y la cubierta vegetal tendrá mayor o menor éxito en la recolonización de los campos abandonados (Ruiz Flaño, 1993; López Bermúdez et al., 1998).

Comparando la humedad media del suelo del periodo de seis meses previos a la toma de cada fotografía del patrón de vegetación de las laderas de $\mathrm{CO}, \mathrm{AL}$ y BE, se obtiene una correlación significativa entre aquella y el ratio\%cubierta vegetal $/ \%$ suelo desnudo (fig. 4). En CO y AM, la relación entre ambas variables fue lineal y el coeficiente de correlación de Pearson significativo (CO: $r^{2}=0.93 ; \mathrm{AL}: r^{2}=0.89 ; p>0.05$ ), mientras que en BE la relación obtenida era de tipo logarítmico $\left(\mathrm{BE}: r^{2}=0.83 ; p>0.05\right)$. La mayor pendiente de la función logarítmica indicaba en el caso de BE, el lugar más semiárido de los tres, una mayor efectividad en el consumo del agua disponible en el

Figura 4. Comparación de la humedad media del suelo y la ratio\%cubierta vegetal/\%suelo desnudo. Abreviaturas: $\mathrm{CO}$, Colmenar; AL, Almogía; BE, Berja; $C V$, cubierta vegetal; SD, suelo desnudo.

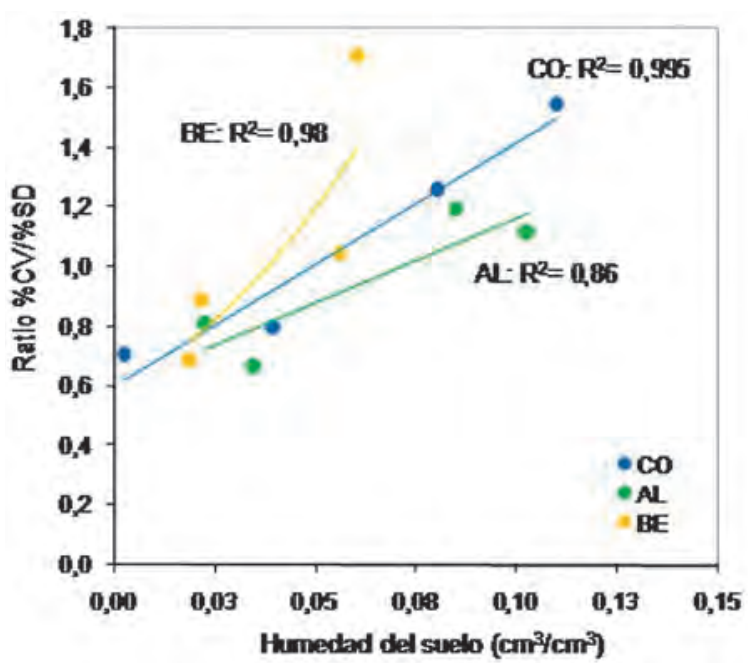


suelo por parte de la vegetación, cuya cubierta permanecía en niveles semejantes a los de $\mathrm{CO}$ y $\mathrm{AL}$ a pesar de las menores precipitaciones.

Después de más de 50 años de abandono, el sistema ecogeomorfológico de las laderas, parece haber evolucionado sujeto al clima, la topografía y las condiciones de degradación del suelo. El clima a través del régimen de precipitaciones limita la disponibilidad de agua para el crecimiento de la vegetación y esta a priori ha sido superior en CO y AL. La topografía, por medio de la pendiente, limita la capacidad de infiltración de los suelos y la retención de agua (Hewlett y Hibbert, 1967; Solé-Benet et al., 1997); todos los campos abandonados presentaban una pendiente elevada (>20\%) con lo que la escorrentía y, por tanto, la erosión hídrica podrían haber estado favorecidos. De hecho, los valores registrados de humedad no eran demasiado elevados, incluso después de eventos lluviosos abundantes. Por último, los suelos de los campos abandonados se caracterizaban por unas condiciones superficiales que corroboraban dichos procesos de erosión hídrica al haber abundante pedregosidad superficial, lo cual indicaba un frecuente proceso de lavado de finos por la escorrentía superficial, especialmente en los campos abandonados de CO y AL, fenómeno observado también por Cerdá (2002).

Respuesta hidrológica del suelo en las lluvias simuladas. La tabla 4 recoge los datos hidrológicos medidos durante las simulaciones de lluvia. Salvo en las parcelas instaladas en matorral, la generación de escorrentía fue generalizada en todas las condiciones superficiales de suelo seleccionadas, aunque se observaron diferencias bastante evidentes en la respuesta hidrológica. En general, hubo una cierta tendencia a registrar más escorrentía en suelos que presentaban una pedregosidad superficial embebida formando una costra continua, así como un pico de sedimentos en supensión más elevada en los campos abandonados con condiciones climáticas más semiáridas.

Como cabía esperar, la presencia de matorral implicó un retraso en el tiempo de encharcamiento y precipitación necesaria para el comienzo de la escorrentía, lo cual era más evidente cuando la pedregosidad superficial se disponía sobre el suelo, no embebida en su superficie. Por otro lado, no observamos diferencias significativas entre el volumen de precipitación necesario para que se iniciara la escorrentía, ni tampoco un efecto significativo de la humedad antecedente del suelo como cabría esperar $(r=-0.38 ; N=25, p<0.05)$.

La tasa de infiltración estable varió significativamente según el tipo de condición superficial del suelo y vegetación. Las tasas más bajas se registraron en las parcelas con una pedregosidad superficial $>70 \%$ y embebida en la superficie, como era característico en $\mathrm{AB}$ y $\mathrm{BE}$; también, son algo bajas en aquéllas con pedregosidad embebida entre un 25 y $70 \%$. No se obtuvo una relación significativa entre la humedad antecedente del suelo y la tasa de infiltración ( $\mathrm{r}=-0.05 ; N=25, p<0.05)$. Es destacable un mayor incremento en la humedad del suelo en algunas parcelas sin cubierta vegetal con pedregosidad superficial dispuesta sobre el suelo (como en CO o AL), en comparación a otras ocupadas bien por matorral dado que el a veces denso sistema aéreo de este retrasaba la llegada de agua y su infiltración en el suelo, o bien por pedregosidad embebida y encostramientos. Una tendencia similar a la tasa de infiltración, se obtuvo al calcular los coeficientes de escorrentía, mayores en los campos abandonados caracterizados por zonas de suelo desnudo, con abundante pedregosidad superficial embebida. 


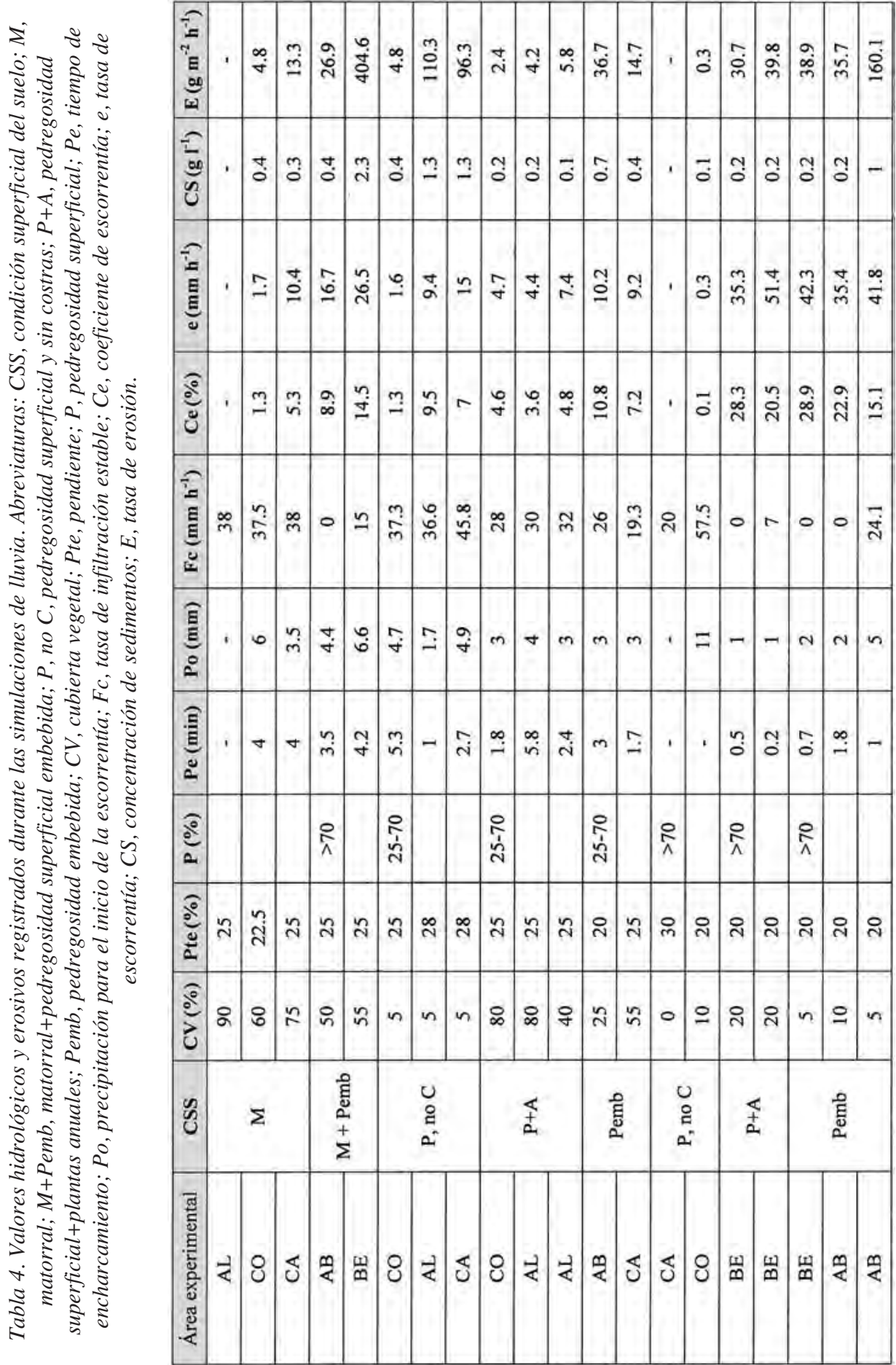


Por último, los datos relativos a la movilización de sedimentos indican dos aspectos relevantes: uno, el efecto positivo de la cubierta vegetal que limita el transporte de sedimentos al ser menos frecuente la generación de escorrentía bajo ella; y otro, el agotamiento en sedimentos de algunas áreas desnudas, pues por ejemplo, parcelas con pedregosidad superficial entre el 25 y $70 \%$, no embebida, emitieron pocos sedimentos lo que parece evidenciar que se encuentran muy lavadas por la más frecuente escorrentía superficial de estas áreas. También, fue menor el arrastre de sedimentos en aquéllas parcelas, especialmente de $\mathrm{CO}$ y AL, de áreas de suelo sin cubierta de matorral (desnudos) pero con presencia de vegetación herbácea anual o terófita.

Los experimentos de lluvia simulada parecen corroborar las hipótesis ya planteadas en apartados anteriores. La cubierta vegetal de los campos abandonados los ha recolonizado en mayor o menor medida dependiendo de las condiciones climáticas, topográficas y edáficas (Kosmas et al., 2000). Estos factores, salvo el climático, parecen ser más restrictivos en los campos bajo condiciones más húmedas ( $\mathrm{CO}$ y $\mathrm{AM}$ ) puesto que la cubierta vegetal no se ha recuperado totalmente después de más 50 años de abandono y la presencia de pedregosidad no embebida implica que ha habido hasta ahora una erosión hídrica más activa que en los campos semiáridos. Sin embargo, actualmente, dicha pedregosidad puede estar protegiendo el suelo de esa erosión hídrica como indican los bajos valores de sedimentos medidos en la escorrentía. Este efecto protector de la pedregosidad superficial ya ha sido observado por otros autores como Poesen y Lavee (1994), Ruiz Sinoga y Martinez Murillo (2009), y Ruiz Sinoga at al., (2010). Por el contrario, en los campos más semiáridos, la pedregosidad suele estar aún embebida, lo cual puede indicar que dicho proceso de lavado así de menor magnitud como resultado de unas precipitaciones más escasas siendo mayor la disponibilidad de finos. Sin embargo, como indican los resultados de las simulaciones, si la cubierta vegetal no sigue aumentando, lo cual es complicado por esa misma falta de precipitaciones, el proceso de lavado continuará y, por tanto, el proceso degradacional del sistema ecogeomorfológico de estos campos.

\section{Conclusiones}

Cinco campos abandonados fueron seleccionados en similares condiciones topográficas, edáficas y de uso actual, pero con diferentes condiciones climáticas, para analizar las semejanzas y diferencias de sus patrones de vegetación y la respuesta hidrológica de sus suelos. Las conclusiones del estudio son las siguientes:

1) Después de más de 50 años de abandono, las áreas experimentales seleccionadas se caracterizaban todas ellas por un patrón de vegetación discontinuo o de dos fases (áreas de vegetación y suelo desnudo), típico de numerosas zonas de media montaña mediterránea con antiguo uso agrícola, incluidas las más lluviosas. Esto indicaba que las condiciones climáticas no habían sido suficientemente eficaces para contrarrestar el deterioro del sistema ecogeomorfológico debido a la antigua actividad agrícola, agravado por la elevada pendiente del terreno que había favorecido la erosión del suelo por la escorrentía superficial. 
2) El resultado fue la definición de unas condiciones superficiales de los suelos caracterizadas por la presencia de abundantes fragmentos rocosos en superficie, indicativo del lavado de finos por la erosión hídrica, proceso que ha sido más eficaz en los campos abandonados con más abundantes precipitaciones. Este proceso degradacional se tradujo en una limitación de la capacidad de retención hídrica de los suelos y, por tanto, de la humedad y agua disponible para la vegetación, que tenía muy limitada su crecimiento, si bien este es más eficaz en los campos abandonados semiáridos, los cuales han conseguido equipar su cubierta vegetal a los más lluviosos.

3) Sin embargo, la falta de una cubierta vegetal continua implica que los procesos de erosión hídrica, de lavado de finos, sean aún más efectivos en los campos abandonados pues las menores precipitaciones no se han traducido en un número suficiente de eventos que lavasen completamente dichos finos. Sí ocurrió así en los campos en condiciones climáticas más húmedas, donde el sistema ecogeomorfológico parece encontrarse en unas condiciones de equilibrio pues, una vez lavados todos los finos, la pedregosidad que ha quedado sobre el suelo, no embebida, parece protegerlo de la erosión hídrica y favorece la infiltración del agua en el suelo, contrarrestando el efecto negativo de la elevada pendiente del terreno.

4) Por tanto, a pesar de que el patrón y cubierta de vegetación de los campos abandonados son similares aún difiriendo en las condiciones climáticas, las condiciones superficiales de sus suelos están indicando estadios diferentes en la evolución de éstos después del abandono de la actividad agrícola: los campos con más abundantes lluvias ( $\mathrm{CO}$ y $\mathrm{AL}$ ) han alcanzado un equilibrio entre sus condiciones climáticas, topográficas y erosivas, mientras que los que están en condiciones más semiáridas aún se encuentran en una fase anterior donde la erosión hídrica puede presentar una elevada actividad durante los escasos eventos lluviosos, como indica la presencia de pedregosidad superficial embebida en superficie, formando una costra continua.

\section{Agradecimientos}

Los autores quieren expresar su agradecimiento al III Plan Andaluz de Investigación y a la Agencia Andaluza del Agua por su apoyo financiero para esta investigación.

\section{Referencias bibliográficas}

Arnau-Rosalen, E., Calvo-Cases, A., BoiX-Fayos, C., Lavee, H., Sarah, P. (2008). Analysis of soil surface component patterns affecting runoff generation. An example of methods applied to Mediterranean hillslopes in Alicante (Spain). Geomorphology, 101: 595-606. 
BergKAmp, G. (1998). A hierarchical view of interactions of runoff and infiltration with vegetation and microtopography in semiarid shrublands. Catena, 33: 201-220.

Bergkamp, G., Cerdè, A., Imeson, A. C. (1999). Magnitude-frequency analysis of water redistribution along a climate gradient in Spain. Catena, 37: 129-146.

Boix, C., Soriano, M. M., Tiemessen, I. R., Calvo, A., Imeson, A. C. (1995). Properties and erosional response of soils in a degraded ecosystem in Crete (Greece). Environmental Monitoring Assessment, 37: 79-92.

Boix, C., Calvo, A., Imeson, A. C., Soriano, M. D., Tiemessen, I. R. (1998). Spatial and short-term temporal variations in runoff, soil aggregation and other properties along a mediterranean climatological gradient. Catena, 33: 123-138.

Boix Fayos, C. (1999). Procesos geomórficos en diferentes condiciones ambientales mediterráneas: el estudio de la agregación y la hidrología de suelos. Tesis doctoral, Servicio de Publicaciones de la Universidad de Valencia, 394 pp., Valencia.

Calvo, A., Boix, C., Imeson, A. C. (2003). Runoff generation, sediment movement and soil water behaviour on calcareous (limestone) slopes of some Mediterranean environments in Southeast Spain. Geomorphology, 50: 269-291.

Calvo, A., Boix, C., Arnau, E. (2005). Patterns and thresholds of runoff generation and sediment transport on some Mediterranean hillslopes. En: Catchment Dynamics and River Processes: Mediterranean and Other Climate Regions (García, C., Batalla, R. J., Ed.). Elsevier, Amsterdam, pp. 31-51.

Castillo, V., Gómez-Plaza, A., Martínez-Mena, M. (2003). The role of antecedent soil water content in the runoff response of semiarid catchments: a simulation approach. Journal of Hydrology, 284: 114-130.

CERDÀ, A. (1998). The influence of geomorphological position and vegetation cover on the erosional and hydrological processes on a Mediterranean hillslope. Hydrological Processes, 12: 661-671.

Cerdà, A., Imeson, A. C., Calvo, A. (1996). Fire and aspects induced differences on the erodibility and hydrology of soils at La Costera, Valencia, SE Spain. Catena, 24: 289-304.

Descroix, L., Gautier, E. (2002). Water erosion in the Southern French Alps: climatic and human mechanisms. Catena, 50: 53-85.

García Ruiz, J. M., Lasanta, T., Gonzáles, C., White, S., Ortigosa, L., Ruiz-Flaño, P. (1995). Changes in runoff and erosion as a consequence of land-use changes in the Central Spanish Pyrenees. Physic and Chemistry of the Earth, 20: 301-307.

Hewlett, J. D., HibBert, A. R. (1967). Factors affecting the response of small watersheds to precipitation in humid areas. En: Forest Hydrology (Sopper, W. E. y Lull, H. W., ed.). Pergamon, pp. 275-290.

Imeson, A. C., LAVEe, H. (1998). Soil erosion and climate change: the transect approach and the influence of scale. Geomorphology, 23: 319-337. 
Imeson, A. C., CAMmeraAt, L. H. (2002). Environmentally sensitive areas in the MEDALUS target areas study sites. En: Mediterranean desertification: A mosaic of processes and responses (Geeson, N.A., Brandt, C.J., Thornes, J. B., Ed.). Wiley \& Sons, Chichester, pp. 177-185.

Imeson, A. C., Lavee, H., Calvo, A., Cerdà, A. (1998). The erosional response of calcareous soils along a climatological gradient in Southeast Spain. Geomorphology, 24: 3-16.

Kosmas, C., Danalatos, N. G., Gerontidis, S. (2000). The effect of land parameters on vegetation performance and degree of erosion under Mediterranean conditions. Catena, 40: 3-17.

Kosmas, C., Danalatos, N. G., López-Bermúdez, F., Romero-Díaz, M. A. (2002). The effect of land use on soil erosion and land degradation under Mediterranean conditions. En: Mediterranean desertification: A mosaic of processes and responses (Geeson, N. A., Brandt, C. J., Thornes, J. B., ed.). Wiley \& Sons, Chichester, pp. 57-70.

Lasanta, T., Pérez Rontomé, C., García-Ruz, J. M., Machín, J., Navas, A. (1995). Hydrological problems resulting from farmland abandonment in semi-arid environments: the Central Ebro Depression. Physic and Chemistry of the Earth, 20: 309-314.

Lasanta, T, García-Ruiz, J. M., Pérez-Rontomé, C., Sancho-Marcén, C. (2000). Runoff and sediment yield in a semiarid environment: the effect of land management after farmland abandonment. Catena, 38: 265-278.

Lavee, H., Poesen, J. (1991). Overland flow generation and continuity on stone cover soil surfaces. Hydrological Processes, 5: 345-360.

Lavee, H., Imeson, A. C., Sarah, P., Benjamini, Y. (1991). The response of soils to simulated rainfall along a climatological gradient in an arid and semiarid region. Catena, 19: 19-37.

LAvee, H., Imeson, A. C., SARAh, P. (1998). The impact of climate change on geomorphology and desertification along a Mediterranean arid transect. Land Degradation and Development, 9: 407-422.

LÓPEZ BERMúdeZ, F. (1993). Reflexiones sobre la degradación de los suelos y su gestión sostenible en la Cuenca mediterránea. En: Desertificación y uso del suelo en la Cuenca Mediterránea. Paralelo 37, 16: 211-218

López Bermúdez, F., Romero Díaz, A., Martínez Fernández, J. (1998). Vegetation and soil erosion under a semiarid Mediterranean climate: a case study from Murcia (Spain). Geomorphology, 24: 51-58.

Martínez Murillo, J. F., Ruiz Sinoga, J. D. (2007). Seasonal changes in the hydrological and erosional response of a hillslope under dry-Mediterranean conditions (Montes de Málaga, South of Spain). Geomorphology, 88: 69-83.

NAREdo, J. M. (1996). La evolución de la agricultura en España (1940-1990), Granada. 
Nicolau, J. M., Solé, A., Puigdefábregas, J., Gutiérrez, L. (1996). Effects of soil and vegetation on runoff along a catena in semi-arid Spain. Geomorphology, 14: 297-309.

OBANDO, J. (2002). The impact of land abandonment on regeneration of semi-natural vegetation: a case study from the Guadalentín. En: Mediterranean desertification: A mosaic of processes and responses (Geeson, N. A., Brandt, C. J., Thornes, J. B., ed.). Wiley \& Sons, Chichester, pp. 269-276.

Poesen, J., Ingelmo, F. (1992). Runoff and sediment yield from topsoils with different porosity as affected by rock fragments. Catena, 19: 451-474.

Poesen, J., LAvee, H. (1994). Rock fragments in topsoils: significance and processes. Catena, 23: 1-28.

Poesen, J., Van Wesemael, B., Bunte, K., Solé-Benet, A. (1999). Variation of rockfragment cover and size along semiarid hillslopes: a case-study from southeast Spain. Geomorphology, 23: 323-335.

Puigdefábregas, J., Del Barrio, G., Boer, M. M., Gutiérrez, L., Solé, A. (1998). Differential responses of hillslope and channel elements to rainfall events in a semiarid area. Geomorphology, 23: 337-351.

Puigdefábregas, J., Solé, A., Gutiérrez, L., Del Barrio, G., Boer, M. (1999). Scales and processes of water and sediment redistribution in drylands: results from the Rambla Honda field site in Southeast Spain. Earth-Science Reviews, 48: 39-70.

Ruiz-Flaño, P. (1993). Procesos de erosión en campos abandonados del Pirineo. Geoforma Ediciones, 220 pp., Logroño.

Ruiz Sinoga, J. D., Martínez Murillo, J. F. (2009). Effects of soil surface components on soil hydrological behavior in a dry Mediterranean environment (Southern Spain). Geomorphology, 108: 234-245.

Ruiz Sinoga, J. D., Romero Díaz, A., Ferré Bueno, E., Martínez Murillo, J. F. (2010). The role of soil surface conditions in regulating runoff and erosion processes on a metamorphic hillslope (Southern Spain) Soil surface conditions, runoff and erosion in Southern Spain. Catena, 80: 131-139.

Solé Benet, A., Calvo, A., Cerdà, A., Lázaro, R., Pini, R., Barbero, J. (1997). Influences of micro-relief patterns and plant cover on runoff related processes in badlands from Tabernas (SE Spain). Catena, 31: 23-28.

Topp, G. C., Davis, J. L., Annan, A. P. (1980). Electromagnetic determination of soil water content: Measurements in coaxial transmission lines. Water Resources Research, 68: 574-582.

Vachaud, G., Passerat de Silans, A., Balabanis, P., Vauclin, M. (1985). Temporal stability of spatially measured soil water probability density function. Soil Science Society of America Journal, 49: 822-828. 
VALENTín, C. (1994). Surface sealing as affected by various rock fragment covers in West Africa. Catena, 23: 87-97.

Van Wesemael, B., Poesen, J., Kosmas, C. S., Danalatos, N. G., Nachtergaele, J. (1996). Evaporation from cultivated soils containing rock fragments. Journal of Hydrology, 182: 65-82.

YAIR, A., KLEIN, Y. (1973). The influence of surface properties on flow and erosion processes on debris covered slopes in an arid area. Catena, 1: 1-14.

Yair, A., LAvee, H. (1985). Runoff generation in arid and semi-arid zones. Hydrological Forecasting, 133: 433-446.

YAIR, A., ENZEL, Y. (1987). The relationship between annual rainfall and sediment yield in arid and semiarid areas. The case of the northern Negev. Catena, 10: 121-135. 